\title{
A Systematic Review of the Evidence for Family and Parenting Interventions in Low- and Middle-Income Countries: Child and Youth Mental Health Outcomes
}

\author{
Gloria A. Pedersen $\mathbb{1}^{1,2,4} \cdot$ Eva Smallegange $^{3} \cdot$ April Coetzee $^{3} \cdot$ Kim Hartog $^{3} \cdot$ Jasmine Turner $^{3} \cdot$ Mark J. D. Jordans $^{2,3} \cdot$ \\ Felicity L. Brown ${ }^{3}$
}

Published online: 29 April 2019

(c) The Author(s) 2019

\begin{abstract}
Objectives Children and youth in low-and middle-income countries (LMIC) are at greater risk for poor mental health. Adverse circumstances including poverty, violence, and lack of available psychological treatments increase their vulnerability. Given the importance of the family environment for child and youth wellbeing, family interventions are a powerful mode of treatment; however, their development and evaluation has received relatively little attention in LMIC.

Methods This review presents evidence for family- and parent-focused interventions on mental health outcomes for children and youth in LMIC and identifies treatment components present in promising interventions. A systematic search was conducted using comprehensive search terms in five databases (Global Health, PubMed, PsychINFO, PILOTS, and Cochrane Library). Reporting follows PRISMA guidelines. Independent raters screened and retrieved articles for inclusion, completed quality ratings, conducted data extraction, and coded common practice elements.

Results This review included 36 papers representing 32 unique studies of family or parenting interventions in LMIC. Study designs covered: RCTs (50\% of studies), pre-to-post studies (38\%), and other (12\%). The majority of interventions showed positive outcomes for child and youth mental health and wellbeing. The two most frequently used treatment techniques were caregiver psychoeducation and caregiver coping skills; the next most common were treatment processes of providing between-session homework and accessibility promotion.

Conclusions Evidence for family-focused interventions for child and youth mental health in LMIC is growing with several promising approaches that should be more rigorously evaluated. Further research into effects of specific intervention components will ensure targeted and optimally effective interventions.
\end{abstract}

Keywords Child $\cdot$ Youth $\cdot$ Family $\cdot$ Psychosocial interventions $\cdot$ Low- and middle-income countries

Supplementary information The online version of this article (https:// doi.org/10.1007/s10826-019-01399-4) contains supplementary material, which is available to authorized users.

Gloria A. Pedersen gapedersen@gwu.edu

1 London School of Hygiene and Tropical Medicine, London, UK

2 King's College London, London, UK

3 War Child Holland, Research and Development, Amsterdam, The Netherlands

4 Present address: The George Washington University, 2120 L St NW, Suite 600, Washington, DC 20037, USA
Globally, mental health concerns are one of the leading causes of lost productivity in young people (Mokdad et al. 2016). Children and youth under the age of 24 years old account for $42 \%$ of the world's population, and roughly $90 \%$ of these live in low- and middle-income countries (LMICs) (Kieling et al. 2011; United Nations 2017). Living in LMICs, children and youth have increased vulnerability to mental illness and the associated high mortality rates due to their social and environmental circumstances, such as increased rates of poverty, violence, and lack of service providers and available medical and psychological treatment (Lund et al. 2018; Patel et al. 2016). The majority of mental disorders have their onset during childhood or youth (Kessler et al. 2007), yet can persist and result in negative effects throughout the lifespan. A gap in research and 
implementation of effective and evidence-based support in LMICs still exists, leaving large numbers of children and youth without access to quality care (Brown et al. 2017; Knerr et al. 2013; Saxena et al. 2007).

Insufficient capacity within mental health workforces presents a major barrier for access to quality care in LMICs (Saxena et al. 2007; WHO 2006). Appropriate training, supervision and infrastructure are needed to provide professional care, but in LMICs there is commonly a lack of funding and availability of trained specialists to provide such services (Saraceno et al. 2007; Saxena et al. 2007). Lower-cost mechanisms have been introduced and are increasingly showing effectiveness. For example, tasksharing, a collaborative model of care whereby nonspecialists are trained to deliver interventions with supervision from specialists, has demonstrated feasibility and effectiveness for increasing the coverage of general health, and mental health services (Patel 2012; Patel et al. 2009).

Additionally, transdiagnostic approaches, that is, interventions utilising common treatment techniques that can be delivered across various diagnostic categories or presenting difficulties, have demonstrated significant potential in treating common mental disorders (Patel et al. 2016) and have been effectively delivered at the community level through non-specialists (Patel et al. 2007). Such approaches have the potential to reduce training needs amongst lay providers and reach more individuals efficiently, as it reduces the requirement for diagnostic processes and training in multiple diagnosis-specific packages. However further research into effective implementation and scale up of such approaches is needed.

Several recent systematic reviews have indicated promising results for the feasibility and effectiveness of using evidence-based psychological intervention approaches from high-income settings in LMICs, to address mental health of children and adults across different cultures, and often via non-specialist delivery models (Brown et al. 2017; Jordans et al. 2016; Knerr et al. 2013; Singla et al. 2017; Tol et al. 2011). Several limitations are identified in the existing evidence base, including the need for more rigorous evaluation trials, systematic processes for cultural and contextual adaptations, further research to understand the effective components of intervention packages, and costeffective methods for training and supervision.

To address the mental health and wellbeing of children and youth, consideration of the family environment is vital, given the powerful impact this has on the child. A positive caregiver-child relationship and nurturing family environment are essential for healthy child development (Biglan et al. 2012). Protective factors for child and youth mental health include family-related signals such as parent mental health, family cohesion, and parent-child attachment (Patel et al. 2008; White et al. 2014). Risk factors for poor mental health include caregiver ill mental health such as depression or substance abuse, and aggressive family environments or violence (Parsons et al. 2012; Repetti et al. 2002).

Further, in LMICs, there is an increased prevalence of other social and environmental risk factors which can impact children and youth directly, or indirectly via the family environment. These risk factors include armed conflict, community violence, poverty, and prevalence of serious health concerns such as positive HIV status (Lund et al. 2011; Miller and Jordans 2016). Exposure to armed conflict can have detrimental effects on mental health and wellbeing, for both children and adults (Dimitry 2012; Lund et al. 2018). A recent systematic review on predictors of household interpersonal violence in humanitarian settings found that exposure to conflict and political violence is associated with increased rates of violence towards women and children (Rubenstein et al. 2017). Therefore, armed conflict has a direct impact on a child's mental health through traumatic exposures (Chrisman and Dougherty 2014; Tamashiro 2010), as well as indirect effects via various mechanisms including increased family violence (Repetti et al. 2002). Positive HIV status has been found to be associated with higher rates of common mental disorders including depression, in both children and adults (Abas et al. 2014; Chibanda et al. 2016). Furthermore, parental positive HIV status also impacts on offspring, including fear of transmission interrupting and limiting parent-child interaction, and disclosure stigma affecting outside social support (Kidia et al. 2015; RAND Health 2009). Therefore, HIV within the family can have a significant impact on a child, regardless of their own HIV status.

Overall, utilizing the powerful influence of parent/caregivers and family members in interventions for child and youth mental health outcomes (Panter-Brick et al. 2014), combined with advances in scalable non-specialist and transdiagnostic approaches, has potential to provide lowcost, high-impact support for children and youth in LMICs where resources are low and risk for poor mental health is high.

Given these complex factors, and in order to develop adequate interventions to address the mental health of young people in LMICs, it is important to understand the evidence base for what has been tested, where, and for whom. There have been several recent systematic reviews considering psychosocial interventions for child and youth mental health outcomes in LMICs (Jordans et al. 2016; Purgato et al. 2018; Tol et al. 2013). In terms of parenting interventions, reviews conducted to date in LMICs have been specific in focus, for example, interventions for children with developmental disorders (Hastings et al. 2012), interventions for improving parenting skills and addressing abusive parenting in LMICs (Knerr et al. 2013) and parenting programmes focusing on early child development 
(ECD) (Britto et al. 2015). The recently increasing evidence base in this field is encouraging, however, only one recent systematic review has considered parenting and familyfocused psychosocial interventions more broadly for all children and youth in LMICs (Healy et al. 2018)

While evidence for the effectiveness of a given intervention package within a given population provides important information for practitioners and policy-makers, an understanding of the 'active ingredients' of effective interventions would provide essential information for further developing and implementing targeted and efficient interventions that can be delivered across settings. This is particularly important given the heterogeneity in interventions identified through reviews (Brown et al. 2017; Jordans et al. 2016; Knerr et al. 2013; Singla et al. 2017; Tol et al. 2011, 2013) and the need for flexible transdiagnostic intervention packages in LMICs to reduce training and supervision needs. The most rigorous way to determine the effective components of an intervention would be through specific research designs such as dismantling studies or measuring different outcomes at multiple timepoints. This kind of research is often not possible or prioritized in LMICs and humanitarian contexts where resources are low, access to care is limited, and the impetus to provide the best available care to all participants is high.

One method that aims to identify important practice elements within interventions is the distillation and matching approach (Chorpita et al. 2005). In this approach, the 'practice elements' across intervention manuals are identified using a structured coding manual and systematic process (distillation), and then data mining techniques are used to determine specific profiles of common elements used in effective treatments, and whether these profiles differ across particular diagnostic groups or settings (matching). While this approach cannot identify which components are the active mechanisms, it does provide more detailed information that may inform future research. This method has been applied to child and adult psychological interventions more broadly (Chorpita et al. 2005; Jordans et al. 2011). Brown et al. (2017) conducted a systematic review of psychological interventions for youth affected by armed conflict and applied the distillation step to identify common practice elements present in those interventions demonstrating an effect.

Given the broad range of approaches to parent/caregiver and family interventions, understanding the elements that constitute family-focused treatment "packages" in LMICs is particularly important. This can inform future dismantling of studies whereby intervention components are individually trialled and compared, alongside mediational analyses to identify potential mechanisms of action, and will further assist in identifying the active ingredients of such treatments.
The current systematic review outlines the evidence of parent- or family-focused interventions for child and youth mental health and well-being in LMICs, as well as present the first analysis of common practice elements amongst interventions showing a positive effect. The analysis of common practice elements will help to identify the common elements most frequently used within effective parenting and family-focused psychosocial interventions in LMICs. The output of this systematic review will inform the broader academic and practitioner community, as well as inform development of meaningful interventions with potential for significant impact.

\section{Method}

The conduct of this study was informed by the Preferred Reporting Items for Systematic Review and Meta-Analysis (PRISMA) guidelines (Shamseer et al. 2015), the Cochrane Handbook for Systematic Reviews of Interventions (Cochrane 2011) and the Centre for Reviews and Dissemination's guide for undertaking reviews in health care (CRD 2009). The completed PRISMA 2009 Checklist can be found in the online Appendix. The review has been registered on PROSPERO, registration CRD42017067108.

\section{Search Strategy}

A systematic literature search was conducted on the following databases: Global Health (Ovid), PubMed (Ovid), PsychINFO (Ovid), PILOTS (ProQuest), and Cochrane Library (Wiley Online Library). Search terms and list of keywords is shown in Supplementary Table 1S. The Boolean operator "OR" was used to find articles with one or more search terms and synonyms, and "AND" to combine the different concepts for relevant articles. Entry of search terms varied depending on the database platform. The majority were searched by keyword anywhere in the text, except for PubMed which was limited to searching only in the Title/Abstract in order to return relevant results. All databases searched years 1967 - 2017, except for Global Health, in which case the databases earliest year within Ovid was 1973.

Truncation and wildcards were used to account for UK and US spelling and terminology. The three main concepts combined included Population AND Intervention AND Outcome. For example, families AND "middle income countr*" AND "parenting intervention" AND "mental health."

Study design was not included in the search terms, but instead was considered during the inclusion/exclusion process. Randomized controlled trials (RCTs), cluster RCTs, stepped wedge designs, non-randomised controlled trials, 
and pre-to post studies were included. We included other study types as we expected relatively few RCTs to be identified and aimed to provide a comprehensive overview of current evidence available and approaches tested, rather than conclusively determine effects of interventions.

Hand searches through selected reference lists of the identified articles and related reviews were conducted in order to account for any relevant references that may have been missed in the original search. Additionally, the authors of articles included in this review were contacted via email with a request to inform us of any additional published studies which met our criteria.

\section{Study selection}

The database search results were exported to EndNote X8, which was also used to remove duplicates. A charting form was created and used to identify eligible studies to be included in the review according to inclusion and exclusion criteria in Supplementary Table 2S. Initial inclusion/exclusion ratings were made by the first author (GP) based on review of the title and abstract only. For increased reliability, these ratings were then confirmed by a second rater. Studies found to be potentially relevant based on the title and abstract were retrieved, and full text was reviewed by the first author (GP), confirmed by a second rater, with any discrepancies being resolved by a third rater (FB).

\section{Eligibility criteria}

Eligibility criteria were specified according to the PICO model (Population, Intervention, Comparison, Outcome), and in line with PRISMA guidelines (Shamseer et al. 2015) (see Table 2S). Intervention studies published between years 1967-2017, in any language, were considered. Studies must have involved a parenting or family intervention, delivered in a LMIC, included quantitative data at a baseline and endline assessment (with or without a comparison group), and assessed improvements in youth mental health or wellbeing. For the purpose of this review, child or youth is defined as any person younger than age 24 (UNESA 2010). Parenting-focused intervention is defined as any programme involving a parent/caregiver, which aims to improve outcomes for the children and parent/caregiver. For example, it may address parenting skills, relationship building, or managing one's own emotions in context of parenting. Family-focused intervention is defined as any programme involving parents or caregivers and/or the family unit which aims to improve outcomes for members of the family by improving family engagement, relationships, and effectiveness in handling adversity (poverty, disorder or illness, daily stressors, disaster, conflict, etc.). Family was broadly defined, and not limited to biological or immediate family members. LMIC was defined according to the World Bank List. Where a study was conducted in a country that is now classified as a HIC but was classified as a LMIC in the five years preceding the publication date, it was included.

\section{Data extraction}

A data extraction sheet was designed in Excel to support the extraction of relevant and detailed information including the author, year of publication, context, study design, intervention type, and study details (i.e. sample, therapist type, sessions, outcomes, etc.). The data extraction was performed by the first author (GP) and was then confirmed by a second rater. Any discrepancies were resolved by a third rater $(\mathrm{FB})$. A final check of data presented in the review was conducted as the last step.

\section{Quality assessment}

The included studies were rated on their methodological quality using a version of the NIH Quality Assessment Tool for Before-After (Pre-Post) Studies With No Control Group (NIH 2014), adapted to include additional questions for RCTs from the Jadad Scale for Reporting Randomised controlled Trials (Halpern and Douglas 2005). The adapted version of the table and its criteria is displayed in Supplementary Tables $3 \mathrm{~S}-4 \mathrm{~S}$. Initial ratings were conducted by the first author (GP), then checked by a second rater with any discrepancies resolved by a third rater (FB).

\section{Data analysis}

In line with the primary objective, a narrative synthesis was conducted using content analysis. Content analysis involves tabulating the frequency of each theme or measure within the categories to determine key findings (Snilstveit et al. 2012). Studies were classified into two subgroups according to the style of the intervention (parent/caregiver focused, or family focused) and characteristics of the studies are summarised, including all outcomes, measures used, and any significant findings.

\section{Coding of Practice Elements}

Interventions that demonstrated positive effects through any research methodology were included in an analysis of practice elements. For permission and access to treatment manuals used in the included studies, authors of the study or the developers of particular treatments the authors used were written requests via email, which included two followup requests. When access was provided, and the manuals were identified as the exact protocols used for the treatment, 
they were used for coding of the practice elements along with any relevant journal articles. In the case access was unavailable, the description and protocol written in relevant journal articles was used $(n=6)$.

Two clinical psychologists (ES and FB) independently coded treatment elements; any discrepancies were resolved by a third rater (MJ). Treatment coding was guided by the PracticeWise coding system (Chorpita et al. 2005), along with an additional code "homework," which is selected from a coding system for common elements for evidencebased trauma treatment (Strand et al. 2013).

Where additional elements were identified that were either not covered in the Practice Wise Coding system, or where further differentiation of these specific techniques was seen to be valuable given the aims of this review, 'other' codes were created. These additional codes were reviewed by both researchers at the end of the coding process, and the most commonly coded, or most important for informing research and practice, were selected for systematic coding across all manuals. In this case, two additional sets of codes were identified. One set of codes considered common topics that were addressed, which may be of particular relevance for LMICs, which included: alcohol and drugs, grief and loss, financial topics, building family identity, HIV, and stigma. Another set of codes were those which specified elements of positive parenting that were more detailed than those included in the Practice Wise coding system: affection, rules and setting limits, realistic expectations about child development, and routines.

As a further step that was considered relevant for coding family interventions in particular, some codes were broken down into sub-codes which specified one or more provider and beneficiary of the technique: facilitator delivered to child; facilitator delivered to parent; facilitator taught parent to deliver to child.

\section{Results}

\section{Study Selection}

A total of 930 papers were retrieved from the search in the five databases. An additional 15 were retrieved manually through hand-searching, and 6 were retrieved from emailing authors of included papers for additional studies. After exporting to EndNote and removing duplicates, 826 articles were prepared for record screening of title and abstracts. After screening, 724 papers were excluded, leaving 101 to go under full-text review. This resulted in a final 36 articles to be included in the data extraction stage. Reasons for exclusion of the other 65 studies included: programme only looking at a parent's own psychological well-being, programme only looking at child cognitive variables, only physical outcomes, programmes working primarily with the child and involve parents in adjunctive sessions (i.e. school sessions), parents or family with offspring aged 25 years or older, single case studies, qualitative data only, not a psychosocial intervention, not an intervention study, families living in high-income countries (HICs), studies without pre or post quantitative data, or it was the wrong population. There were two papers in Mandarin which were excluded due to inability to obtain a thorough English translation in the allotted time.

The 36 papers included in the data extraction stage reported on 32 unique studies-quantitative results from two studies were reported across two papers each. The PRISMA diagram (Fig. 1) illustrates the selection process.

\section{Study Characteristics}

\section{Study locations and settings}

All studies took place in a LMIC as defined by the World Bank List (World Bank 2017) thereby providing a range of settings. According to the WHO regional classification (WHO 2017), studies took place in Africa $(n=14)$, the Americas $(n=5)$, Southeast Asia: $(n=2)$, Europe: $(n=4)$, Eastern Mediterranean: $(n=2)$ and the Western Pacific $(n$ $=5$ ). Most studies were conducted in a community setting $(n=22)$ for example a public school or a community organization, other settings included primary healthcare $(n$ $=3)$, mental healthcare $(n=4)$, other hospital setting $(n=$ 1) (i.e. a government hospital), and other settings $(n=2)$ (i.e. home visits).

\section{Study design}

Interventions were categorized as either parent-focused or family-focused. A total of 14 studies identified in this review were categorized as parent-focused. In some cases, children were present, but in any case, they were not directly involved in the programme as far as the therapy, training, or education. All studies of parent-focused interventions measured at least one mental health outcome or benefit for the child/youth of the parent/caregiver. Study designs varied within this category and involved using RCTs $(n=7)$, non-randomised controlled trial $(n=3)$, and pre-to post design $(n=4)$. A total of 18 studies identified in this review were categorized as family-focused. All studies included at least one outcome for the child/youth, though not every study assessed outcomes for the parent/caregiver and/or family member. Study designs varied within this category and included RCTs $(n=9)$, pre- to post $(n=8)$, and other (stepped wedge; $n=1$ ) 
Fig. 1 The PRISMA Flow Diagram of Study Selection Process. http://prisma-statement. org/PRISMAStatement/FlowDia gram.aspx

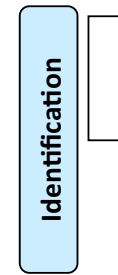

Records identified through database searching $(n=930)$

Additional records identified through other sources $(n=21)$
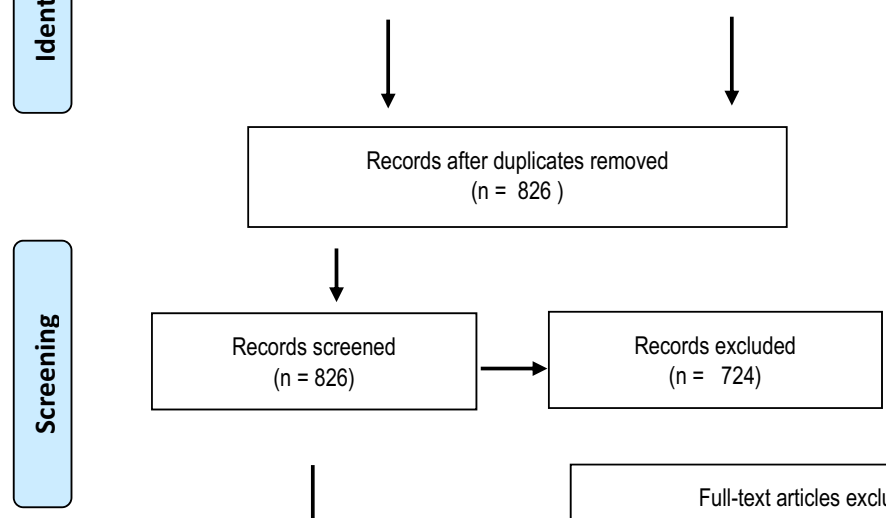

Full-text articles excluded: $(n=65)$

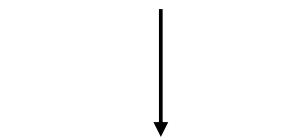

Reasons:

Program only looking at parent's own psychological wellbeing:

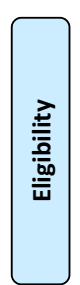

Full-text articles assessed for eligibility $(n=101)$

Parents or family with members aged 25 years or older $\mathrm{n}=14$

Programs that work primarily with the child and involve parents in adjunctive sessions (i.e. school sessions) $\mathrm{n}=1$

Program only working with the child (i.e. anger management, cognitive rehab, etc.) $\mathrm{n}=2$

Only child cognitive variable (i.e. attention, executive

Studies excluded in data extraction stage $(n=2)$

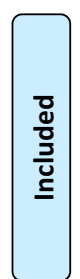

Reasons:

Unable to translate in allotted time

Studies included in narrative synthesis $(n=36)$ functioning, impulsivity) $\mathrm{n}=2$ No child mental health outcomes $\mathrm{n}=2$

Only physical outcomes $n=1$

Wrong population $n=4$

Single case studies $\mathrm{n}=3$

Studies w/o pre-and post-quantitative outcome data $n=7$

Not an intervention study $\mathrm{n}=13$

Not a psychosocial intervention $\mathrm{n}=4$

Families living in high-income countries $\mathrm{n}=2$

\section{Study quality}

The study quality was rated on a "Good," "Fair," or "Poor" scale to determine introduced bias. Papers were rated "Good" ( $n=16)$ if 10 or more (out of 12) quality rating criteria were answered "yes," and rated "fair" $(n=15)$ or "poor" $(n=1)$ if three or more criteria were not met ("no") or difficult to determine ("cannot determine" or " 0 " for RCT subscale). Many studies failed to report whether or not people assessing the outcomes were blinded to the participants' exposures/interventions $(n=8)$. Several studies also proved difficult to determine whether the study population was a sufficient sample size, lacking report on the statistical power of the study or how they came to the sample size ( $n$
=13). Quality assessment criteria can be found in Supplementary Tables $3 \mathrm{~S}-4 \mathrm{~S}$.

\section{Parent-Focused Interventions}

\section{Target groups}

All studies $(n=14)$ involved at least one parent/caregiver, the majority of which were mothers or grandmothers, while six studies targeted mothers. Ages of children ranged from 2 weeks to 18 years. The majority of studies included children $(n=9)$, three included children and adolescents, and two targeted infants only. A few studies targeted youth with high levels of behavioural problems or difficulties, 
some of which were confirmed through prior diagnoses and reconfirmed through screening in the study (i.e. obsessive compulsive disorder, OCD (Abedi and Vostanis 2010); attention deficit hyperactivity disorder, ADHD (Matos et al. 2006); or autism spectrum disorder, ASD (Bello-Mojeed et al. 2016). Other studies identified behavioural problems through initial screening in the study (Jordans et al. 2013; Yu et al. 2015), or through parental report (Guo et al. 2016; Mejia et al. 2015).

\section{Intervention type used}

A range of parenting interventions were used. Six studies used a parent/caregiver-training and skills building format, three of which used versions of the Triple $\mathrm{P}$ Parenting Programme. Others used psychoeducation $(n=4)$, different kinds of behavioural therapy $(n=3)$, or Quality of Life therapy $(n=1)$. Most interventions focused on better parenting practices to increase mental well-being for the parent/ caregiver, child/youth, and to better family relationships. For example, using a psychoeducational programme specifically aiming to reduce negative parenting practices and its effects on children's behavioural problems (SolisCamara et al. 2000). Of the two studies considering infants, one study focused on early-childhood development and relationship building, including the mental well-being of the parent/caregiver and child (Davis et al. 2005; Puura et al. 2012), and one (Yousafzai et al. 2014, 2015) targeted social-emotional outcomes through a responsive stimulation and nutrition programme.

\section{Format and number of sessions}

Interventions varied in format and number of sessions held. Most sessions were carried out in groups of parents/caregivers $(n=7)$, some used a combination of groups and individual parents/caregivers sessions $(n=4)$ (two in which the child/youth was present), and others were solely individual parents/caregiver sessions $(n=3)$, all of which the child/youth was present. Total number of sessions ranged from 1-25 meetings. Usually, individual sessions were carried out if the topic required significant attention, for example examining parent-child interaction (Davis et al. 2005; Guo et al. 2016; Puffer et al. 2015; Puura et al. 2012; Yousafzai et al. 2014, 2015) and/or the child having significant behavioural or mental difficulties (Matos et al. 2006; Yu et al. 2015).

\section{Implementation factors}

Six studies used mental health specialists including psychologists or training psychologist $(n=4)$, psychiatrist $(n$ $=1$ ), and trained nurses $(n=1)$; while eight of the studies used non-specialists or lay health workers $(n=5)$ or accredited Triple $\mathrm{P}$ facilitators $(n=3)$. Details of service providers' training was mentioned in more than half of the studies $(n=9)$, but only six studies reported details of the supervision provided, three of which were the Triple $\mathrm{P}$ Parenting interventions. Most studies mentioned delivering the intervention in the respective country's mother tongue through translation of materials $(n=2)$, with some making other and/or additional cultural adaptations such as local examples or terminology (Guo et al. 2016; Jordans et al. 2016; Puffer et al. 2015; Sumargi et al. 2015), though reported detail of the processes was limited for most.

\section{Outcomes}

A variety of outcomes were measured on and reported by both parents/caregivers and children/youth amongst the studies with a wide range of results. Table 1 displays each study with all outcome measures collected, along with denotation of significant findings.

\section{Parents/caregivers}

Parenting practices and behaviours such as positive reinforcement, coercive parenting, reduced harsh discipline, and parenting consistency were assessed in seven studies. Parent-child and family relationships and family social support were also assessed in seven studies, as were caregiver mental health and quality of life factors including stress, self-esteem, and general life satisfaction.

\section{Children and youth}

Behavioural problems (including aggression, hyperactivity, obsession, compulsion, and general difficulties) was the most commonly assessed outcome $(n=13)$. Other child/ youth reported measured outcomes included measures of internalizing and externalizing symptoms such as anxiety, depressive symptoms, and stress $(n=7)$, and overall quality of life $(n=1)$.

\section{Family-Focused Interventions}

\section{Target groups}

Seven family-focused interventions involved more than one offspring per family unit,, while 11 involved only one. Parent/caregiver type varied throughout the studies, though two targeted mothers for participation. Parent/caregivers ranged and were not limited to natural birth mothers and/or fathers, but also grandparents, aunt, uncle or foster parents. All parents/caregivers were over the age of 18 years. All interventions reported on child/youth outcomes, and 14 also 


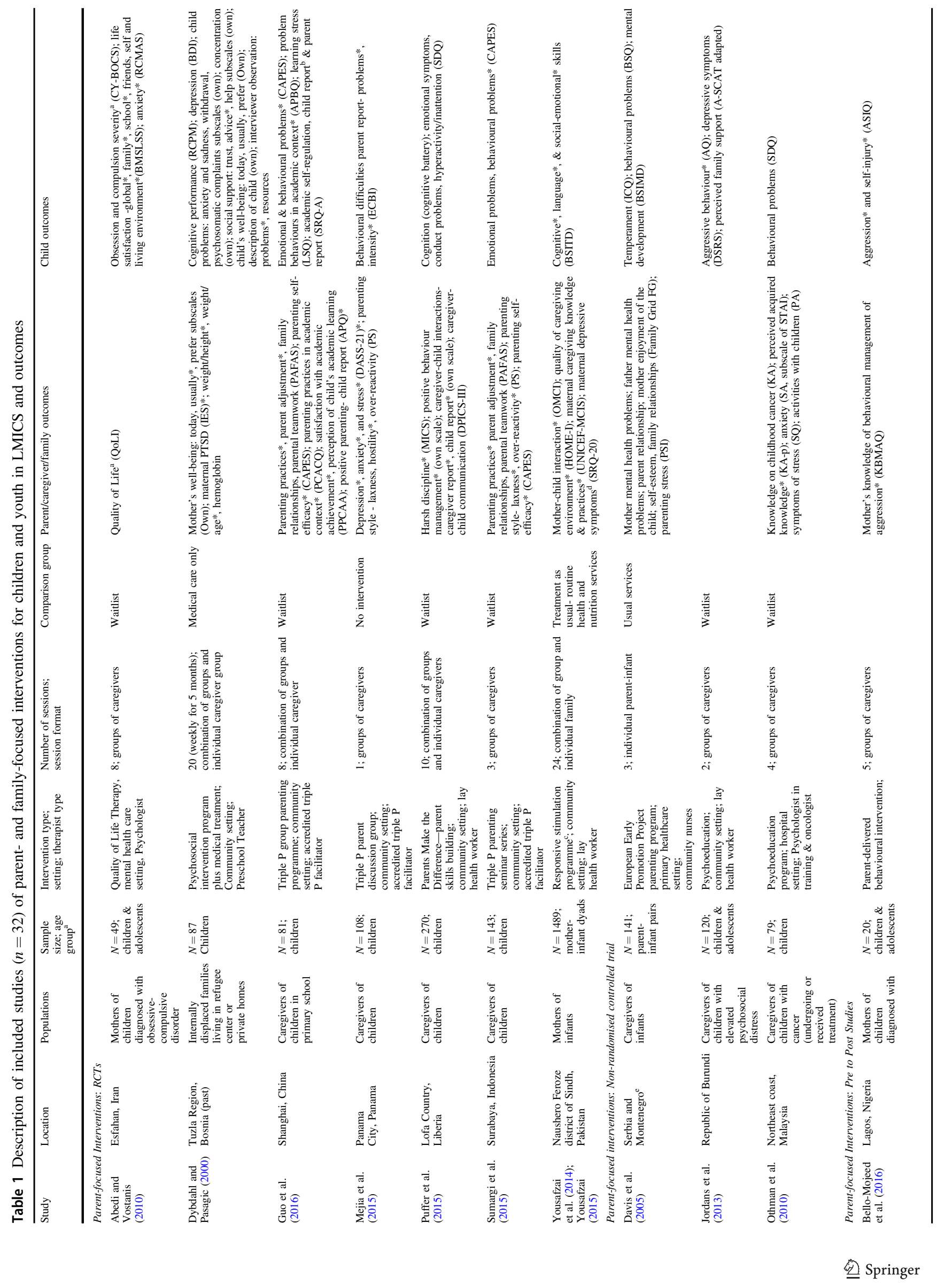




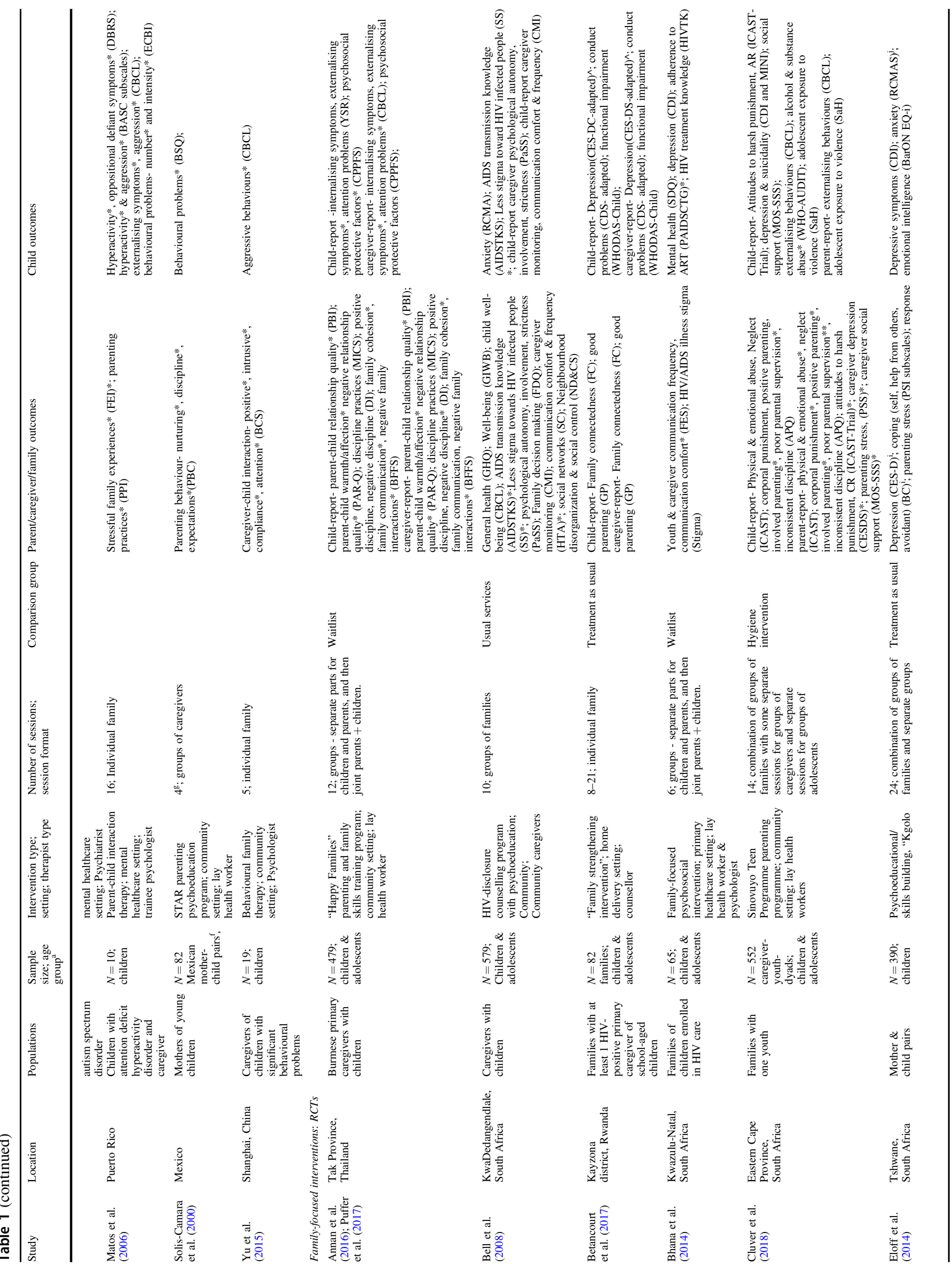




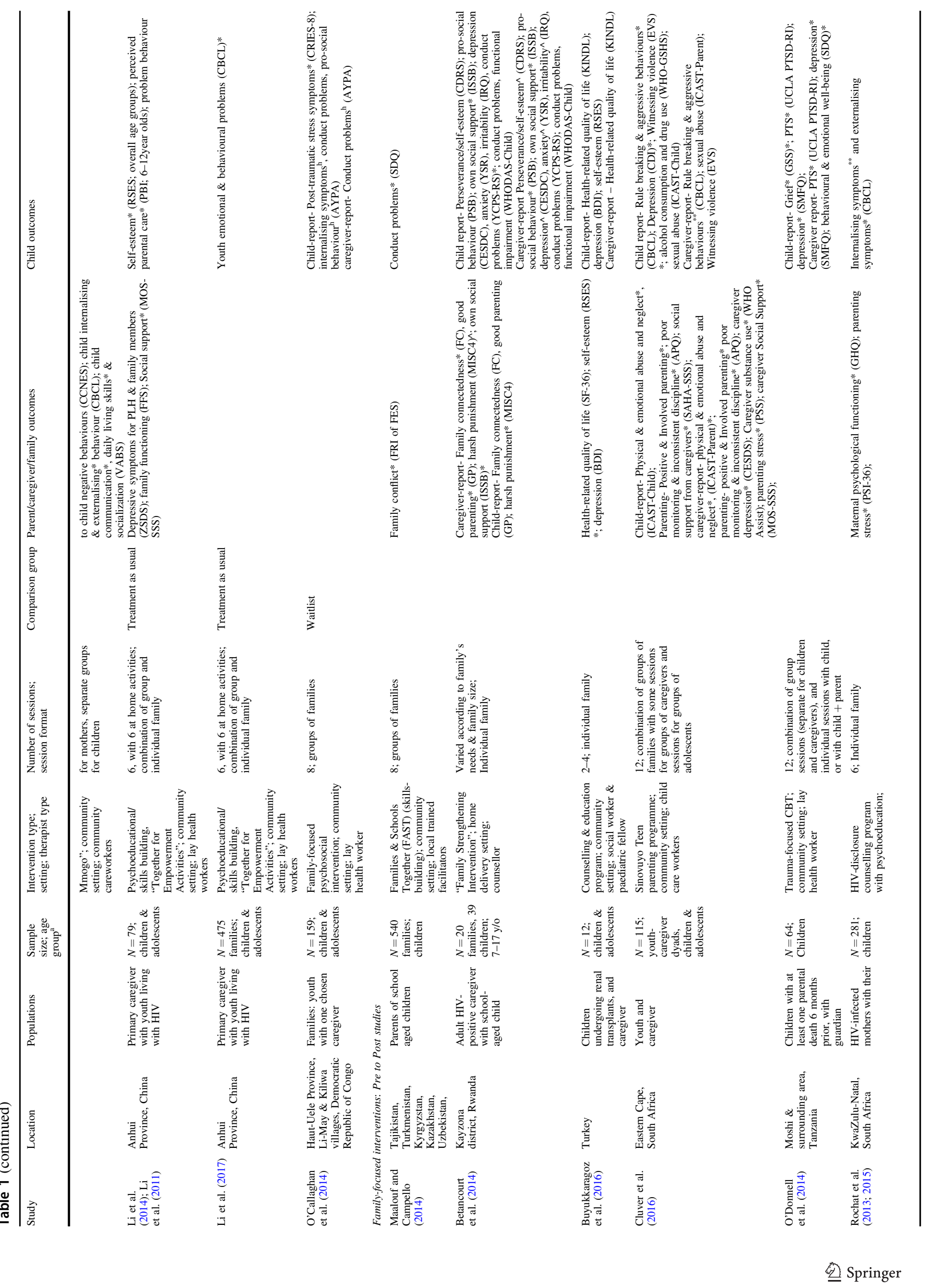




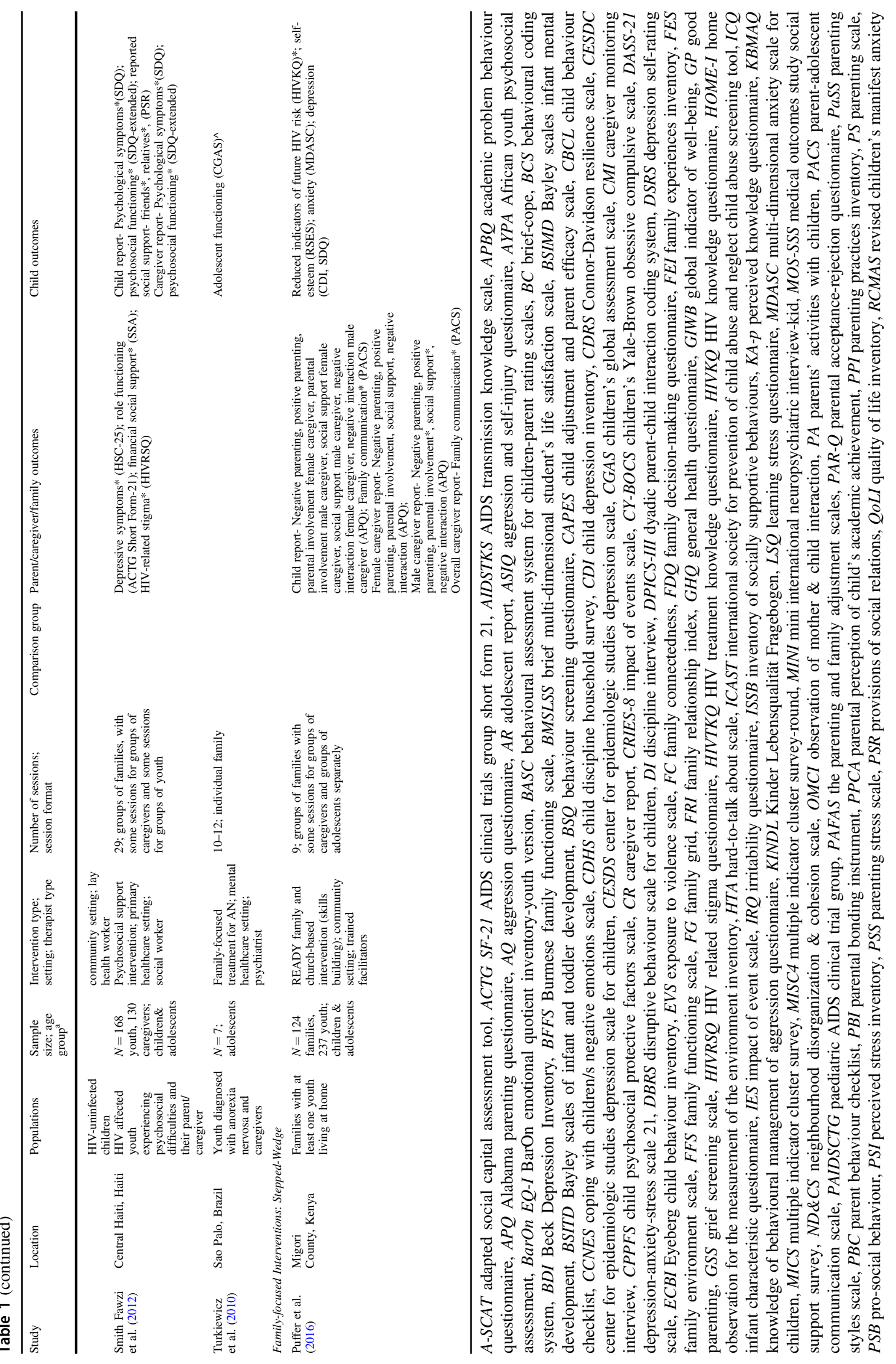


reported on parent/caregiver or family outcomes. Overall child/youth age range was 2-18 years, but age ranges included across studies varied- 13 studies included children and adolescents, 4 studies included children only, and 4 studies included adolescents only. Seven studies targeted one or more participants affected by HIV, which could be the child or youth $(n=2)$, the child or youth and parent/ caregiver $(n=1)$, or the parent/caregiver only $(n=4)$. A few studies $(n=3)$ were designed for specific, lifethreatening illnesses/treatments experienced by the child/ youth such as anorexia nervosa $(\mathrm{AN})$, cancer, or renal transplant. Other interventions focused on groups at-risk for depression, anxiety, post-traumatic stress (PTS), violence, or with HIV-related risks $(n=8)$.

\section{Intervention type used}

A range of interventions were used, with types including psychosocial $(n=3)$, psychoeducational and/or skills building $(n=9)$, counselling with psychoeducation $(n=3)$, trauma-focused cognitive behavioral therapy (CBT) $(n=1)$ and $\mathrm{AN}$-focused treatment $(n=1)$. Many interventions were conducted in community settings $(n=13)$, with some selecting the setting specifically to encourage participation in the intervention, for example Puffer et al. (2016) delivered the family intervention through churches as most community members attended church.

\section{Format and number of sessions}

Formats of interventions varied, being delivered to groups of families ( $n=10 ; 6$ of which had some separate sessions for children and caregivers), a combination of group and individual family sessions $(n=3)$, and solely individual family sessions $(n=5)$. The average number of sessions ranged from 4-24 meetings, though one study used a combined 29 sessions for the family over two phases (Smith Fawzi et al. 2012).

\section{Implementation factors}

Most studies used non-specialists, including lay health workers ( $n=10 ; 1$ which was assisted by a psychologist), facilitators $(n=2)$, and child care workers $(n=1)$; while four studies used specialists or paraspecialists including social workers $(n=2)$ and counsellors $(n=2)$, and one study used a psychiatrist $(n=1)$. Training was mentioned in 14 of the studies, though not always with detail on length or strategy. Supervision was mentioned in 12 of the studies. 11 studies reported on conducting the intervention in the community's mother tongue. Though not always in addition to local language delivery, 15 studies mentioned in small detail other cultural adaptations including local role models, 
expressions, and examples (Annan et al. 2016; Betancourt et al. 2014, 2017; Bhana et al. 2014; Cluver et al. 2016; Cluver et al. 2018; Eloff et al. 2014; Li et al. 2014; Maalouf and Campello 2014; O'Callaghan et al. 2014; O'Donnell et al. 2014; Puffer et al. 2016; Rochat et al. 2015; Smith Fawzi et al. 2012).

\section{Outcomes}

A variety of outcomes were measured for children/youth, parent/caregivers, and family followed by a wide range of results. Table 1 displays each study with all outcome measures collected, along with denotation of significant findings.

\section{Family and parent/caregiver outcomes}

Family outcomes such as connectedness, functioning, and family or parent-child communication were the most commonly assessed $(n=8)$. Parent/caregiver mental healthrelated outcomes were also assessed $(n=8)$ including depressive symptoms, and stress, as well as social support $(n=8)$, and health-related quality of life $(n=1)$. Experience of HIV illness stigma was measured in three studies. Parenting practices was another measure assessed $(n=7)$, with studies looking at good parenting, levels of harsh punishment, child monitoring, and managing one's anger when dealing with one's child.

\section{Child and youth outcomes}

Over the 18 studies, 30 outcomes were measured and assessed. The most common outcomes were internalizing symptoms $(n=14)$ such as anxiety, depression, grief and PTS, and externalizing symptoms $(n=13)$ such as conduct problems. Functioning was also assessed $(n=5)$, along with social support $(n=3)$, physical, emotional or sexual abuse $(n=2)$, alcohol and substance use $(n=2)$, overall quality of life $(n=1)$ and HIV-knowledge and indicators of HIV risk $(n=3)$.

\section{Common Practice Elements Analysis (Chorpita et al. 2005) for Parent-focused and Family-focused Interventions}

Of the total of 32 studies in the review, 28 intervention protocols were coded from studies which showed a positive effect for child or youth mental health and wellbeing outcomes in LMICs (Chorpita et al. 2005). In three cases, identical intervention manuals were used in multiple studies, and were only included once (Betancourt et al. 2017; Betancourt et al. 2014; Cluver et al. 2016; Cluver et al. 2018; Li et al. 2017; Li et al. 2014). One study only showed intervention effects in HICs (though LMICs were included in the study (Davis et al. 2005; Puura et al. 2012) and therefore this intervention manual was not included in the coding process). In five studies, positive effects were seen only for parenting outcomes (parent self-esteem (Buyukkaragoz et al. 2016), parenting practices (Puffer et al. 2015), parent communication (Bell et al. 2008; Bhana et al. 2014) and parenting knowledge (Othman et al. 2010). It was decided to retain these manuals for analysis, given the potential for improvements in parenting outcomes to lead to beneficial effects for children. These 25 intervention manuals were coded on the 72 Practice Wise codes, plus the additional code of Homework. They were also coded on the topics addressed, and specific positive parenting techniques (see methods). Thirty practice elements were identified in at least 8 of the coded manuals, and these are displayed in Fig. 2. The remainder of the practice elements were identified in 7 or less manuals and therefore not presented. As shown in Fig. 2, the most common practice elements, occurring in more than $50 \%$ of the intervention manuals were: psychoeducation for caregivers, caregiver coping, accessibility promotion, homework, praise, support networking, insight building, relationship/rapport building, activity scheduling, communication skills, goal setting, maintenance and relapse prevention, and modelling. Notably, caregiver psychoeducation was present in all manuals $(n=28)$. Specific topics covered in interventions included: HIV $(n=8)$, such as medication adherence and psychoeducation; stigma $(n=7)$; finances $(n=4)$; alcohol and other drugs $(n=3)$; and family identity $(n=10)$. Additional positive parenting techniques that were identified were: giving affection $(n=$ 14); setting rules and limits $(n=12)$; maintaining realistic expectations of the child and their development $(n=10)$; and establishing clear routines $(n=10)$.

\section{Discussion}

This review sought to identify the evidence for parent- and family-focused interventions for child and youth mental health and well-being outcomes in LMICs. A total of 36 papers were found, representing 32 studies on a range of interventions in a variety of LMIC. By organising the results into categories, this review was able to conduct a narrative synthesis on 14 parent-focused interventions and 18 familyfocused interventions. Results demonstrated that parent- and family-focused interventions (i.e. psychoeducation, parentand family-skills training, behavioural, psychosocial, and trauma-focused-CBT) may be beneficial to LMIC populations, as 28 studies (88\%) showed a significant positive effect in the intervention group on a myriad of outcomes including child and youth mental health and wellbeing, as well as parenting behaviours and family functioning. 


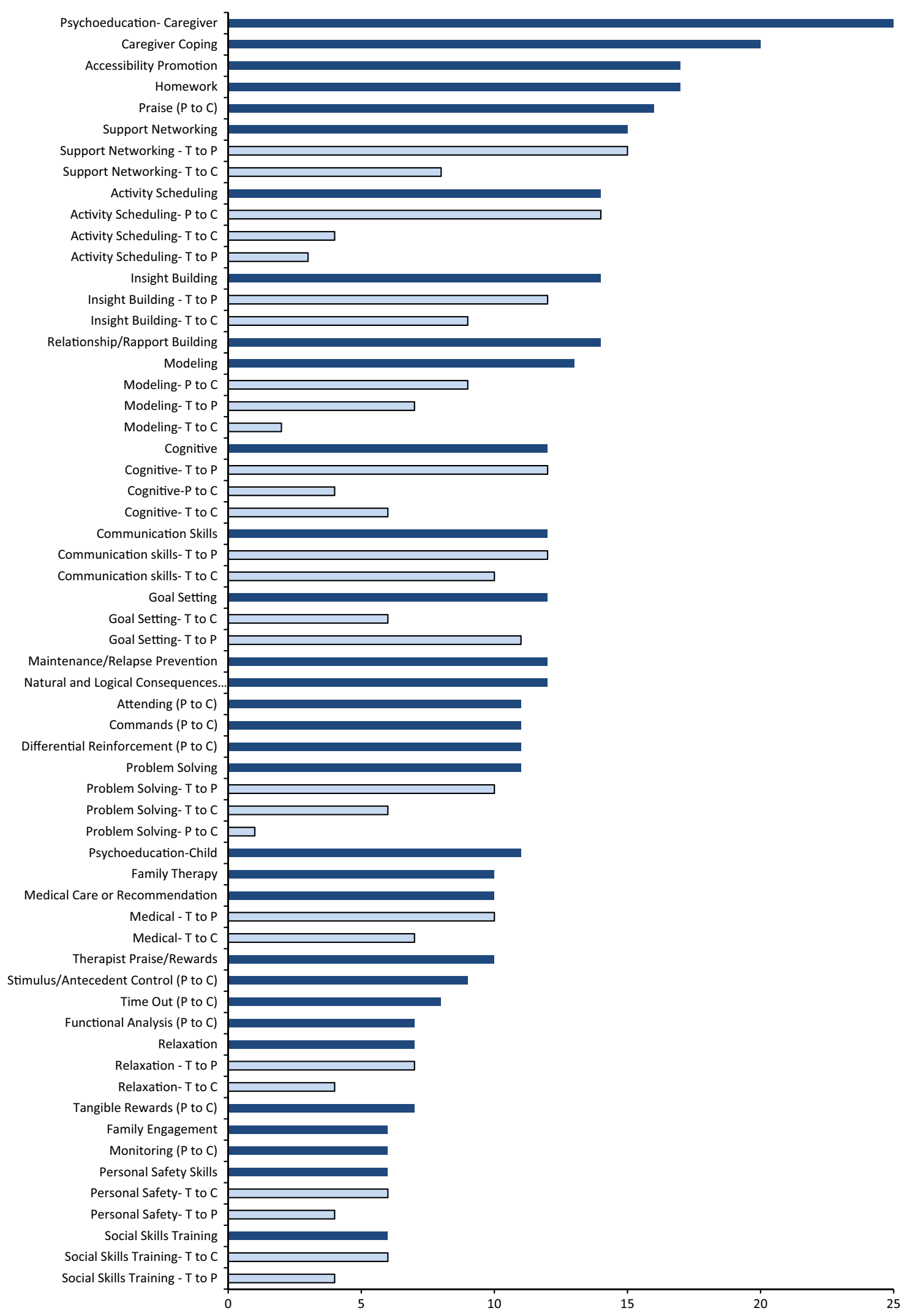

Fig. 2 Practice element profile $^{1}$ for studies of family or parenting interventions $(n=25)$ showing significant improvements in child or caregiver outcomes in LMICs. ${ }^{1}$ Coded according to PracticeWise to parent, $\mathrm{P}$ to $\mathrm{C}$ therapist teaches parent to deliver to child. Darker lines refer to overall code, lighter lines refer to codes by target and (2005) coding system. Note. T to C therapist to child, T to P therapist 
This review found that over half of all included studies $(65 \%)$ used non-specialists (e.g., lay health workers, facilitators, or child worker) to deliver the intervention, where others used mental health specialists (i.e. psychiatrist, psychologist; 19\%) or trained paraspecialists (i.e. social worker, counsellor, nurses; 16\%). RCTs or cluster RCTs were used in roughly $50 \%$ of studies, pre-to post design in $38 \%$, and study designs such stepped wedge and nonrandomised controlled trials in the other $13 \%$. Lack of rigour in study conduct was prominent, with $50 \%$ of included studies rated as "Good." Particularly amongst the 16 RCTs, only 6 received maximum rating for full reporting on the process of randomization, blinding, and account of all participants. Overall, sample sizes were generally small, with close to half of the studies having less than 100 participants included, and with about the same proportion failing to report the power of the sample size, indicating less than favourable risk of bias throughout. Knerr et al. (2013) reported on interventions for positive parenting skills in LMICs and reported the same limitation -only 2 of the 12 included papers had large sample sizes with low risk of bias.

Family and parent-focused interventions in this review addressed a variety of populations within LMIC, including communities affected by armed conflict (Jordans et al. 2013; O'Callaghan et al. 2014), higher rates of violence and violent behaviours (Maalouf and Campello 2014), or high risk of or being affected by HIV (Bell et al. 2008; Eloff et al. 2014; Puffer et al. 2016), to specific clinical groups including autism spectrum disorder and obsessive compulsive disorder (Abedi and Vostanis 2010; Bello-Mojeed et al. 2016).

The most common practice elements included strategies delivered to caregivers (e.g. caregiver coping skills), parenting strategies delivered to caregivers to implement with children/youth (e.g. praise, activity scheduling for the family, modelling behaviours) and strategies that could be delivered to both caregivers and children/youth (e.g. support networking, activity scheduling, insight building, cognitive strategies, and communication skills). They also included elements related to the delivery of the intervention such as promoting access, assigning homework between sessions, and specifically discussing maintenance of changes. Therapeutic elements of relationship and rapport building, and therapist praise or rewards, were also specifically noted in many manuals.

\section{Methodological Limitations}

There was a high level of heterogeneity between studies, which limits the ability to draw strong conclusions from the narrative synthesis and to compare and contrast findings from different studies. Some studies were very specific in their aim, targeting niche groups with particular problems (i.e. OCD or cancer), whereas others targeted larger groups with more general commonality such as geographical area or shared religious beliefs, adding further variation in the interventions. Given these results, it was not possible to adequately analyse potential context-dependent differences. This limitation is comparable to those reported in similar systematic reviews (Brown et al. 2017). Due to the exploratory nature of the review, and the heterogeneous nature of treatment samples, intervention types, and outcomes, a meta-analysis was deemed inappropriate. Two articles written in Mandarin were unable to be translated within the time allotted, and therefore were excluded from this review. Also, the study search was limited to published literature only, and cannot report on any grey literature that may provide further information.

\section{Recommendations for Future Research}

This review supports prior reports, such as by Knerr et al. (2013) that parenting interventions improve positive parenting behaviour, and that low-cost mechanisms for delivery of psychosocial interventions can be effectively implemented in adverse LMIC contexts (Brown et al. 2017; Jordans et al. 2016; Singla et al. 2017; Tol et al. 2011). More importantly, this review adds to the evidence base by highlighting the positive effects on child and youth mental health and wellbeing outcomes from both parent- and family-focused interventions. Recommendations for future research are:

This analysis does not provide evidence for the causal relation between use of specific practice elements and outcomes, as it only provides us with insight into commonly used elements within effective packages. Future research therefore should begin to investigate which components of interventions and implementation characteristics are individually effective, requiring dismantling studies or mediational analyses of mechanisms of change. Identification of active components could aid appropriate allocation of the limited available resources within LMICs to carry out targeted and cost-effective interventions. This would also help to facilitate the scale-up of such parenting and family programmes, a major goal and repeated struggle seen within the field.

Attention should also be paid to common therapeutic factors (i.e. communication skills, engagement) which are seen as universal practices that contribute to the effectiveness of any intervention (Barth et al. 2011). Though the PracticeWise coding system includes some, such as relationship and rapport building, and therapist praise and rewards, they are not always specified in intervention manuals, nor captured in the delivery of RCTs when published, and may be missed. Furthermore, as mounting evidence from HICs shows that common therapeutic factors 
play a significant role in therapeutic outcome (Lambert and Kleinstauber 2016), competence in these is particularly important to consider when training non-professionals in LMICs. An 18-item tool to measure lay counsellor competence in common factors when delivering interventions in LMICs has been developed and recently evaluated (Kohrt et al. 2015; Kohrt et al. 2019). For researchers conducting future trials, measurement and reporting of therapist competence and use of common factors through such tools, and direct observations of sessions, would provide valuable information. Additionally, detailed reporting on the extent to which these common factors were covered in training protocols would be important.

Only 17 of the 32 studies in this review conducted follow-up assessments. Preferably studies should include follow-up assessments to determine long-term impact of interventions to determine whether any initial change is maintained. These assessments may also highlight important changes which may occur later in time and might go undetected without longer follow-up. This is particularly relevant when studying parenting and family interventions which aim to have longer term (developmental) outcomes for youth. For example, Puffer et al. (2015) evaluated the "Parents Make the Difference" (PMD) intervention in a post-conflict setting (Liberia) and included a one-month follow-up assessment. PMD aims to provide parent/caregiver skills training in order to increase positive parenting behaviours and child-parent interaction with the expectation it will eventually have a positive effect on the child's overall cognitive, emotional and/or behavioural well-being. No significant effect was identified at either time point for the child, though significant effect was measured at both time points for the parent/caregiver, indicating a longer followup assessment for the child may be necessary to capture long-term effects the intervention may have. For instance, three of the studies in this review found positive, significant changes at the follow-up assessment (Eloff et al. 2014; O'Callaghan et al. 2014; Othman et al. 2010; Turkiewicz et al. 2010) when initial post-intervention collection had shown no difference or no change.

With regards to the mode of delivery of interventions, future research should thoroughly report the implementation details including training, supervision and cultural adaptations. Proper training and supervision helps to ensure fidelity of the programme. Cultural adaptation processes have been identified as necessary for intervention acceptability and effectiveness, but currently the field lacks consensus and evidence on efficient processes that should be followed (Shehadeh et al. 2016). As contexts and resources vary immensely across different settings, documentation and reporting of what type of training, supervision, and adaptations were successful throughout the study helps future researchers to plan for potential barriers and strategize ways to overcome them (Collins et al. 2011; Eaton et al. 2011; Patel et al. 2009). Often during research studies, available resources and expertise for training and supervision are enhanced and this can lead to inflated estimates of effects of interventions that may not be replicable in standard ongoing implementation. Researchers reporting more thoroughly on such important implementation factors will enable more accurate interpretation of results. Additionally, studies implementing interventions with realistic implementation factors from the outset will result in more valuable insight with regards to implementation in daily practice and support for the development of sustainable and scalable solutions. For example, a recent systematic review on implementation and delivery of family interventions by non-specialist providers summarises the importance of using local trainers and supervisors (rather than researchers from HIC) to support sustainable intervention model (Healy et al. 2018)

In LMIC, children and youths' mental health is at an increased risk due to extreme levels of poor social and environmental circumstances such as poverty and violence. An estimated 535 million children are living in countries affected by humanitarian crises, and 230 million are affected by armed conflict-both of which are demonstrated to have negative mental health effects lasting into adulthood (Miller and Jordans 2016; Ventevogel et al. 2013) and are more likely to occur in LMICs (UNICEF 2017). Previous systematic reviews have synthesized the literature for children and youth in LMIC, showing the potential for psychological treatments delivered by non-specialists. To date, family- and parenting-focused interventions have been underrepresented, even with strong evidence for the importance of parents and the family setting in increasing or reducing the risk of child and youth mental illness (Lund et al. 2018; Patel et al. 2016; Tol et al. 2013).

This review retrieved 32 studies of parent- and familyfocused interventions in LMIC. Results demonstrate feasibility and promising results for this mode of intervention within psychoeducational, parent- and familyskills training, behavioural, psychosocial, and traumafocused-CBT delivery on child and youth mental health and wellbeing outcomes. Twenty-eight intervention manuals from the included studies were coded for the intervention elements that they contain, and this revealed that strategies to address caregiver knowledge and personal coping, as well as promote access, engagement, and support, were most commonly used. The review is characterized by the heterogeneity of interventions and low quality of studies. Future studies should involve; (a) conducting rigorous and methodologically sound studies, (b) identifying effective components of interventions, (c) implementation factors such as training, supervision, and cultural adaptation that are associated with improved outcomes. 
Author Contributions GAP and FLB conceptualized the review and wrote the protocol, and MJDJ contributed to revision of the protocol. GAP conducted the searches, screening, performed the quality assessment, performed extraction for reviews and drafted results of the narrative subsections. JT, AC, KH, ES and FLB were second and third raters for the screening, quality assessments and extraction. FLB and ES performed coding of practice elements, with MJDJ as the third rater, and FLB drafted coding elements results subsections. GAP registered the protocol and drafted the manuscript. All authors contributed to the revision of the manuscript.

\section{Compliance with Ethical Standards}

Conflict of Interest The authors declare that they have no conflict of interest.

Ethical Approval This article does not contain any studies with human participants or animals performed by any of the authors.

Publisher's note: Springer Nature remains neutral with regard to jurisdictional claims in published maps and institutional affiliations.

Open Access This article is distributed under the terms of the Creative Commons Attribution 4.0 International License (http://crea tivecommons.org/licenses/by/4.0/), which permits use, duplication, adaptation, distribution, and reproduction in any medium or format, as long as you give appropriate credit to the original author(s) and the source, provide a link to the Creative Commons license, and indicate if changes were made.

\section{References}

References included in the review are marked by an*

Abas, M., Ali, G. C., Nakimuli-Mpungu, E., \& Chibanda, D. (2014). Depression in people living with HIV in sub-Saharan Africa: time to act. Tropical Medine \& International Health, 19(12), 1392-1396. https://doi.org/10.1111/tmi.12382.

*Abedi, M., \& Vostanis, P. (2010). Evaluation of quality of life therapy for parents of children with obsessive-compulsive disorders in Iran. European Child and Adolescent Psychiatry, 19(7), 605-613. https://doi.org/10.1007/s00787-010-0098-4. http:// onlinelibrary.wiley.com/o/cochrane/clcentral/articles/350/CN00759350/frame.html.

*Annan, J., Sim, A., Puffer, E. S., Salhi, C., \& Betancourt, T. S. (2016). Improving mental health outcomes of burmese migrant and displaced children in Thailand: a Community-Based Randomized Controlled Trial of a Parenting and Family Skills Intervention. Prevention Science. https://doi.org/10.1007/s11121-016-0728-2.

Barth, R. P., Lee, B. R., Lindsey, M. A., Collins, K. S., Strieder, F., Chorpita, B. F., \& Sparks, J. A. (2011). Evidence-based practice at a crossroads: the timely emergence of common elements and common factors. Research on Social Work Practice. https://doi. org/10.1177/1049731511408440.

*Bell, C. C., Bhana, A., Petersen, I., McKay, M. M., Gibbons, R., Bannon, W., \& Amatya, A. (2008). Building protective factors to offset sexually risky behaviors among black youths: a randomized control trial. Journal of the National Medical Association, 100(8), 936-944.

*Bello-Mojeed, M., Ani, C., Lagunju, I., \& Omigbodun, O. (2016). Feasibility of parent-mediated behavioural intervention for behavioural problems in children with Autism Spectrum Disorder in Nigeria: A pilot study. Child and Adolescent Psychiatry and Mental Health, 10(28), 10.
*Betancourt, T. S., Ng, L. C., Kirk, C. M., Brennan, R. T., Beardslee, W. R., \& Stulac, S., et al. (2017). Family-based promotion of mental health in children affected by HIV: a pilot randomized controlled trial. Journal of Child Psychology and Psychiatry, 58 (8), 922-930. https://doi.org/10.1111/jcpp.12729.

*Betancourt, T. S., Ng, L. C., Kirk, C. M., Munyanah, M., Mushashi, C., \& Ingabire, C., et al. (2014). Family-based prevention of mental health problems in children affected by HIV andAIDS: an open trial. AIDS, 28(Suppl 3), S359-S368. https://doi.org/10. 1097/qad.0000000000000336.

*Bhana, A., Mellins, C. A., Petersen, I., Alicea, S., Myeza, N., \& Holst, H., et al. (2014). The VUKA family program: Piloting a family-based psychosocial intervention to promote health and mental health among HIV infected early adolescents in South Africa. AIDS Care, 26(1), 1-11.

Biglan, A., Flay, B. R., Embry, D. D., \& Sandler, I. N. (2012). The critical role of nurturing environments for promoting human wellbeing. Am Psychol, 67(4), 257-271. https://doi.org/10.1037/a 0026796.

Britto, P. R., Ponguta, L. A., Reyes, C., \& Karnati, R. (2015). Systematic Review of Parenting Programmes for Young Children. Retrieved from https://www.unicef.org/earlychildhood/files/P_ Shanker_final_Systematic_Review_of_Parenting_ECD_Dec_ 15_copy.pdf.

Brown, F. L., de Graaff, A. M., Annan, J., \& Betancourt, T. S. (2017). Annual Research Review: breaking cycles of violence-a systematic review and common practice elements analysis of psychosocial interventions for children and youth affected by armed conflict. Journal of Child Psychology and Psychiatry, 58(4), 507-524.

*Buyukkaragoz, B., Buyan, N., Dogrucan, N., \& Celik, B. (2016). Health-related quality of life of pediatric renal transplant recipientsand their parents: the role of associated factors and clinical counseling. Turkish Journal of Medical Science, 46(5), 1481-1485. https://doi.org/10.3906/sag-1505-18.

Chibanda, D., Cowan, F., Gibson, L., Weiss, H. A., \& Lund, C. (2016). Prevalence and correlates of probable common mental disorders in a population with high prevalence of HIV in Zimbabwe. BMC Psychiatry, 16, 55. https://doi.org/10.1186/s12888016-0764-2.

Chorpita, B. F., Daleiden, E. L., \& Weisz, J. R. (2005). Identifying and selecting the common elements of evidence based interventions: a distillation and matching model. Mental Health Services Research, 7(1), 5-20. https://doi.org/10.1007/s11020005-1962-6.

Chrisman, A. K., \& Dougherty, J. G. (2014). Mass trauma: distasters, terrorism, and war. Child and Adolescent Psychiatric Clinics of North America, 23(2), 257-279. https://doi.org/10.1016/j.chc. 2013.12.004.

*Cluver, L., Meinck, F., Yakubovich, A., Doubt, J., Redfern, A., \& Ward, C., et al. (2016). Reducing child abuse amongst adolescents in low- and middle-income countries: a pre-post trial in South Africa. BMC Public Health, 16(1), 567. https://doi.org/10. 1186/s12889-016-3262-z.

*Cluver, L., Meinick, F., Steinert, J., Shenderovich, Y., Doubt, J., Romero, R. H., et al. (2018). Parenting for Lifelong Health: a pragmatic cluster randomised controlled trial of a noncommercialised parenting programme for adolescents and their families in South Africa. BMJ Global Health. https://doi.org/10. 1136/bmjgh-2017-000539.

Cochrane. (2011). Cochrane Handbook for Systematic Reviews of Interventions. In Higgins, J. P. T. \& Green, S. (Eds.). Cochrane Handbook for Systematic Reviews of Interventions Version 5.1.0 [updated March 2011]. The Cochrane Collaboration, 2011. Available from www.handbook.cochrane.org. 
Collins, P. Y., Patel, V., Joestl, S. S., March, D., Insel, T. R., \& Daar, A. S., et al. (2011). Grand challenges in global mental health. Nature, 475(7354), 27-30. https://doi.org/10.1038/475027a.

CRD. (2009). Systematic Reviews: CRD's guidance for undsertaking reviews in health care. Centre for Reviews and Dissemination, University of York, 2008.

*Davis, H., Dusoir, T., Papadopoulou, K., Dimitrakaki, C., Cox, A., \& Ispanovic-Radojkovic, V., et al. (2005). Child and family outcomes of the european early promotion project. International Journal of Mental Health Promotion, 7(1), 63-78.

Dimitry, L. (2012). A systematic review on the mental health of children and adolescents in areas of armed conflict in the Middle East. Child Care Health Dev, 38(2), 153-161. https://doi.org/10. 1111/j.1365-2214.2011.01246.x.

*Dybdahl, R., \& Pasagic, I. (2000). Traumatic experiences and psychological reactions among women in Bosnia during the war. Medicine, Conflict and Survival, 16(3), 281-290. https://doi.org/ 10.1080/13623690008409525.

Eaton, J., McCay, L., Semrau, M., Chatterjee, S., Baingana, F., \& Araya, R. et al. (2011). Scale up of services for mental health in low-income and middle-income countries. The Lancet, 378, 1592-1603. https://doi.org/10.1016/S0140-6736(11)60891-X.

*Eloff, I., Finestone, M., Makin, J. D., Boeving-Allen, A., Visser, M., \& Ebersohn, L., et al. (2014). A randomized clinical trial of an intervention to promote resilience in young children of HIVpositive mothers in South Africa. AIDS, 28, S347-S357.

*Guo, M., Morawska, A., \& Sanders, M. R. (2016). A randomized controlled trial of group triple $\mathrm{P}$ with Chinese parents in Mainland China. Behavior Modification, 40(6), 825-851.

Halpern, S. H., \& Douglas, M. J. (2005). Jadad scale for reporting randomized controlled trials. In Halpern, S. H. \& Douglas, M. J. (Eds.), Evidence-based Obstetric Anesthesia. Blackwell Publishing Ltd. https://onlinelibrary.wiley.com/doi/pdf/10.1002/ 9780470988343.app1.

Hastings, R. P., Robertson, J., \& Yasamy, M. T. (2012). Developmental disorders in low and middle income countries. Journal of Applied Research in Intellectual Disabilities, 25, 119-134. https://doi.org/10.1111/j.1468-3148.2011.00680.x.

Healy, E. A., Kaiser, B. N., \& Puffer, E. S. (2018). Family-based youth mental health interventions delivered by nonspecialist providers in low- and middle-income countries: a systematic review. Families, System and Health, 36(2), 182-197. https://doi. org/10.1037/fsh0000334.

Jordans, M. J., Pigott, H., \& Tol, W. A. (2016). Interventions for children affected by armed conflict: a systematic review of mental health and psychosocial support in low- and middle-income countries. Current Psychiatry Report, 18(1), 9 https://doi.org/10. 1007/s11920-015-0648-z.

JordansM. J., Tol, W., \& Komproe, I. (2011). Mental health interventions for children in adversity: pilot-testing a research strategy for treatment selection in low-income settings. Social Science \& Medicine, 73(3), 456-466.

*Jordans, M. J., Tol, W., Ndayisaba, A., \& Komproe, I. (2013). A controlled evaluation of a brief parenting psychoeducation intervention in Burundi. Social Psychiatry and Psychiatric Epidemiology, 48(11), 1851-1859.

Kessler, R., Angermeyer, M., Anthony, J. C., de Graff, R., Demyttenare, K., \& GASQUET, I., et al. (2007). Lifetime prevalence and age-of-onset distributions of mental disorders in the World Health Organization's World Mental Health Survey Initiative. World Psychiatry, 6, 168-176.

Kidia, K., Machando, D., Bere, T., Macpherson, K., Nyamayaro, P., \& Potter, L., et al. (2015). 'I was thinking too much': experiences of HIV-positive adults with common mental disorders and poor adherence to antiretroviral therapy in Zimbabwe. Tropical
Medicine \& International Health, 20(7), 903-913. https://doi.org/ 10.1111/tmi.12502.

Kieling, C., Baker-Henningham, H., Belfer, M., Conti, G., Ertem, I., \& Omigbodun, O., et al. (2011). Child and adolescent mental health worldwide: evidence for action. The Lancet, 378(9801), $1515-1525$.

Knerr, W., Gardner, F., \& Cluver, L. (2013). Improving positive parenting skills and reducing harsh and abusive parenting in lowand middle-income countries: a systematic review. Prevention Science, 14(4), 352-363. https://doi.org/10.1007/s11121-0120314-1.

Kohrt, B. A., Jordans, M. J. D., Rai, S., Shrestha, P., Luitel, N. P., \& Ramaiya, M. K., et al. (2015). Therapist competence in global mental health: Development of the ENhancing Assessment of Common Therapeutic factors (ENACT) rating scale. Behaviour Research and Therapy, 69, 11-21. https://doi.org/10.1016/j.brat. 2015.03.009.

Kohrt, B. A., Mutamba, B. B., Luitel, N. P., Gwaikolo, W., Onyango Mangen, P., Nakku, J., et al. (2019). How competent are nonspecialists trained to integrate mental health services in primary care? Global health perspectives from Uganda, Liberia, and Nepal. International Review of Psychiatry. https://doi.org/10. 1080/09540261.2019.1566116.

Lambert, M., \& Kleinstauber, M. (2016). When people change, and its relation to specific therapy techniques and common factors. Verhaltenstherapie, 26, 1-6. https://doi.org/10.1159/000442372.

*Li, L., Liang, L., Lin, C., Lan, C. W., Ji, G., \& Xiao, Y. (2017). Changes in behavioral outcomes among children affected by HIV: Results of a randomized controlled trial in China. Journal of Health Psychology. https://doi.org/10.1177/1359105317746479.

*Li, L., Liang, L. J., Ji, G., Wu, J., \& Xiao, Y. (2014). Effect of a family intervention on psychological outcomes of children affected by parental HIV. AIDS and Behavior, 18(11), 2051-2058. https://doi.org/10.1007/s10461-014-0744-9.

Li, L., Ji, G., Liang, L. J., Ding, Y., Tian, J., \& Xiao, Y. (2011). A multilevel intervention for HIV-affected families in China: Together for Empowerment Activities (TEA). Social Science \& Medicine, 73(8), 1214-1221. https://doi.org/10.1016/j.socscimed. 2011.07.013.

Lund, C., Brooke-Sumner, C., Baingana, F., Baron, E. C., \& Brever, E. (2018). Social determinants of mental disorders and the Sustainable Development Goals: a systematic review of reviews. The Lancet, 5, 357-369.

Lund, C., De Silva, M., Cooper, S., Chisolm, D., Das, J., Knapp, M., \& Patel, V. (2011). Poverty and mental disorders: breaking the cycle in low-income and middle-income countries. The Lancet. https://doi.org/10.1016/S0140-6736(11)60754-X.

*Maalouf, W., \& Campello, G. (2014). The influence of family skills programmes on violence indicators: experience from a multi-site project of the United Nations Office on drugs and crime in low and middle income countries. Aggression and violent behavior, 19(6), 616-624. https://doi.org/10.1016/j.avb.2014.09.012.

*Matos, M., Torres, R., Santiago, R., Jurado, M., \& Rodriguez, I. (2006). Adaptation of parent-child interaction therapy for Puerto Rican Families: a preliminary study. Family Process, 45(2), 205-222.

*Mejia, A., Calam, R., \& Sanders, M. R. (2015). A pilot randomized controlled trial of a brief parenting intervention in low-resource settings in Panama. Prevention Science, 16(5), 707-717. https:// doi.org/10.1007/s11121-015-0551-1. Retrieved from http:// onlinelibrary.wiley.com/o/cochrane/clcentral/articles/745/CN01255745/frame.html.

Miller, K. E., \& Jordans, M. J. (2016). Determinants of children's mental health in war-torn settings: translating research into action. Curr Psychiatry Rep, 18(6), 58 https://doi.org/10.1007/s11920016-0692-3. 
Mokdad, A. H., Forouzanfar, M. H., Daoud, F., Mokdad, A. A., El Bcheraoui, C., Moradi-Lakeh, M., et al. (2016). Global burden of diseases, injuries, and risk factors for young people's health during 1990-2013: a systematic analysis for the Global Burden of Disease Study 2013. The Lancet. https://doi.org/10.1016/S01406736(16)00648-6.

NIH. (2014, 03/2014). Quality assessment tool for before-after (prepost) studies with no control group. https://www.nhlbi.nih.gov/ health-pro/guidelines/in-develop/cardiovascular-risk-reduction/ tools/before-after.

*O'Callaghan, P., Branham, L., Shannon, C., Betancourt, T. S., Dempster, M., \& McMullen, J. (2014). A pilot study of a family focused, psychosocial intervention with war-exposed youth at risk of attack and abduction in north-eastern Democratic Republic of Congo. Child Abuse \& Neglect, 38(7), 1197-1207.

*O'Donnell, K., Dorsey, S., Gong, W., Ostermann, J., Whetten, R., \& Cohen, J. A., et al. (2014). Treating maladaptive grief and posttraumatic stress symptoms in orphaned children in Tanzania: groupbased trauma-focused cognitive-behavioral therapy. Journal of Traumatic Stress, 27(6), 664-671. https://doi.org/10.1002/jts.21970.

*Othman, A., Blunden, S., Mohamad, N., Mohd Hussin, Z. A., \& Jamil Osman, Z. (2010). Piloting a psycho-education program for parents of pediatric cancer patients in Malaysia. PsychoOncology, 19, 326-331. https://doi.org/10.1002/pon.1584.

Panter-Brick, C., Grimon, M. P., \& Eggerman, M. (2014). Caregiverchild mental health: a prospective study in conflict and refugee settings. Journal of Child Psychology and Psychiatry, 55(4), 313-327. https://doi.org/10.1111/jcpp.12167.

Parsons, C. E., Young, K. S., Rochat, T. J., Kringelbach, M. L., \& Stein, A. (2012). Postnatal depression and its effects on child development: a review of evidence from low- and middle-income countries. British Medical Bulletin, 101, 57-79. https://doi.org/ 10.1093/bmb/ldr047.

Patel, V. (2012). Global mental health: from science to action. Harvard Review Psychiatry, 20(1), 6-12. https://doi.org/10.3109/ 10673229.2012.649108.

Patel, V., Araya, R., Chatterjee, S., Chisholm, D., Cohen, A., \& De Silva, M., et al. (2007). Treatment and prevention of mental disorders in low-income and middle-income countries. The Lancet, 370(9591), 991-1005. 10.1016/s0140-6736(07)61240-9.

Patel, V., Chisolm, D., Parikh, R., Charlson, F. J., Degenhardt, L., \& Dua, T., et al. (2016). Addressing the burden of mental, neurological, and substance use disorders: key messages from Disease Control Priorities, 3rd edition. The Lancet, 387, 1672-1685. 10.1016/S0140-6736(15)00390-6.

Patel, V., Flisher, A. J., Nikapota, A., \& Malhotra, S. (2008). Promoting child and adolescent mental health in low and middle income countries. Journal of Child Psychol and Psychiatry, 49 (3), 313-334. https://doi.org/10.1111/j.1469-7610.2007.01824.x.

Patel, V., Goel, D. S., \& Desai, R. (2009). Scaling up services for mental and neurological disorders in low-resource settings. International Health, 1(1), 37-44. https://doi.org/10.1016/j.inhe. 2009.02.002.

Puffer, E. S., Green, E. P., Chase, R. M., Sim, A. L., Zayzay, J., \& Friis, E., et al. (2015). Parents make the difference: a randomizedcontrolled trial of a parenting intervention in Liberia. Global Mental Health (Camb), 2, e15 https://doi.org/10.1017/gmh.2015.12.

*Puffer, E. S., Green, E. P., Sikkema, K. J., Broverman, S. A., Ogwang-Odhiambo, R. A., \& Pian, J. (2016). A church-based intervention for families to promote mental health and prevent HIV among adolescents in rural Kenya: results of a randomized trial. Journal of Consulting and Clinical Psychology, 84(6), 511-525.

*Puffer, E. S., Annan, J., Sim, A. L., Salhi, C., \& Betancourt, T. S. (2017). The impact of a family skills trainingintervention among Burmese migrant families in Thailand: A randomized controlled trial. PLoS ONE, 12(3), e0172611. https://doi.org/10.1371/journa 1.pone. 0172611 .

Purgato, M., van Ommeren, M., Tol, W., \& Barbui, C. (2018). Addressing stress, depression, and anxiety in people exposed to traumatic events in humanitarian settings: a systematic review and meta-analysis of psychosocial interventions. Journal of Psychosomatic Research, 109, $127 \mathrm{https}: / /$ doi.org/10.1016/j.jpsychores.2018.03.122.

Puura, K., Davis, H., Cox, A., Tsiantis, J., Tamminen, T., \& Ispanovic-Radojkovic, V., et al. (2012). The European Early Promotion Project: description of the service and evaluation study. International Journal of Mental Health Promotion, 7(1), 17-31. https://doi.org/10.1080/14623730.2005.9721947.

RAND Health. (2009). How Parental HIV Affects Children: Research Brief. https://www.rand.org/pubs/research_briefs/RB9372/ index 1.html.

Repetti, R. L., Taylor, S. E., \& Seeman, T. E. (2002). Risky families: family social environments and the mental and physical health of offspring. Psychological Bulletin, 128(2), 330-366. https://doi. org/10.1037//0033-2909.128.2.330.

Rochat, T. J., Mkwanazi N., \& Bland R. (2013). Maternal HIV disclosure to HIV-uninfected children in rural South Africa: a pilot study of a family-based intervention. BMC Public Health, 13(147).

*Rochat, T. J., Arteche, A. X., Stein, A., Mitchell, J., \& Bland, R. M. (2015). Maternal and child psychological outcomes of HIV disclosure to young children in rural South Africa: the Amagugu intervention. AIDS, 29(Suppl 1), S67-S79. https://doi.org/10. 1097/qad.0000000000000668.

Rubenstein, B. L., Lu, L. Z. N., MacFarlane, M., \& Stark, L. (2017). Predictors of interpersonal violence in the household in humanitarian settings: A systematic review. Trauma Violence Abuse, 1524838017738724. https://doi.org/10.1177/1524838017738724.

Saraceno, B., van Ommeren, M., Batniji, R., Cohen, A., Gureje, O., \& Mahoney, J. et al. (2007). Barriers to improvement of mental health services in low-income and middle-income countries. The Lancet, 370(9593), 1164-1174. https://doi.org/10.1016/s01406736(07)61263-x.

Saxena, S., Thornicroft, G., Knapp, M., \& Whiteford, H. (2007). Resources for mental health: scarcity, inequity, and inefficiency. The Lancet, 370(9590), 878-889. https://doi.org/10.1016/s01406736(07)61239-2.

Shamseer, L., Moher, D., Clarke, M., Ghersi, D., Liberati, A., \& Petticrew, M., et al. (2015). Preferred reporting items for systematic review and meta-analysis protocols (PRISMA-P) 2015: elaboration and explanation. BMJ, 349, g7647 https://doi.org/10. 1136/bmj.g7647.

Shehadeh, M. H., Heim, E., Chowdhary, N., \& al., e. (2016). Cultural Adaptation of Minimally Guided Interventions for Common Mental Disorders: A Systematic Review and Meta-Analysis. JMIR Mental Health, 3. https://doi.org/10.2196/mental.5776.

Singla, D. R., Kohrt, B. A., Murray, L. K., Anand, A., Chorpita, B., \& Patel, V. (2017). Psychological Treatments for the World: Lessons from Low- and Middle-Income Countries. Annual Review of Clinical Psychology, https://doi.org/10.1146/annurev-.

*Smith Fawzi, M. C., Eustache, E., Oswald, C., Louis, E., Surkan, P. J., \& Scanlan, F., et al. (2012). Psychosocial support intervention for HIV-affected families in Haiti: implications for programs and policies for orphans and vulnerable children. Social science and medicine, 74(10), 1494-1503. https://doi.org/10.1016/j.socscimed.2012. 01.022 .

Snilstveit, B., Oliver, S., \& Vojtkova, M. (2012). Narrative approaches to systematic review and synthesis of evidence for international development policy and practice. Journal of Development Effectiveness, 4(3), 409-429. https://doi.org/10.1080/19439342. 2012.710641.

*Solis-Camara, R., Fox, R. A., \& Nicholson, B. C. (2000). Parenting young children: comparison of a psychoeducational program in 
Mexico and the United States. Early Child Development and Care, 163, 115-124.

Strand, V. C., Hansen, S., \& Courtney, D. (2013). Common elements across evidence-based trauma treatment: discovery and implications. Advances in Social Work, 14(2), 334-354.

*Sumargi, A., Sofronoff, K., \& Morawska, A. (2015). A randomizedcontrolled trial of the triple P-positive parenting program seminar series with indonesian parents. Child Psychiatry and Human Development, 46(5), 749-761. https://doi.org/10.1007/s10578014-0517-8. Retrieved from http://onlinelibrary.wiley.com/o/ cochrane/clcentral/articles/502/CN-01254502/frame.html.

Tamashiro, T. (2010). Impact of conflict on children's health and disability: Background paper (2011/ED/EFA/MRT/PI/35). United Nations Educational, Scientific and Cultural Organization, Published online: UNESDOC, UNESCO Digital Library. https:// unesdoc.unesco.org/ark:/48223/pf0000190712.

Tol, W. A., Barbui, C., Galappatti, A., Silove, D., Betancourt, T. S., Souza, R., et al. (2011). Mental health and psychosocial support in humanitarian settings: linking practice and research. The Lancet. https://doi.org/10.1016/S0140-6736(11)60941-0 and https://doi.org/10.1016/S0140-6736(11)61270-1.

Tol, W. A., Song, S., \& Jordans, M. J. (2013). Annual Research Review: resilience and mental health in children and adolescents living in areas of armed conflict--a systematic review of findings in low- and middle-income countries. Journal of Child Psychology and Psychiatry, 54(4), 445-460. https://doi.org/10.1111/jcpp.12053.

*Turkiewicz, G., Pinzon, V., Lock, J., \& Fleitlich-Bilyk, B. (2010). Feasibility, acceptability, and effectiveness of family-based treatment for adolescent anorexia nervosa: an observational study conducted in Brazil. Revista Brasileira de Psiquiatria, 32 (2), 169-180.

UNESA. (2010). World programme of action for youth. New York: United Nations.

UNICEF. (2017). UNICEF Humanitarian Action for Children. https://www.unicef.org/publications/files/HAC_2017_Overview_ ENG.pdf.
United Nations. (2017). World Population Prospects: The 2017 Revision, Key Findings and Advance Tables. United Nations, Department of Economic and Social Affairs, Population Division.

Ventevogel, P., Jordans, M., Eggerman, M., van Mierlo, B., \& PanterBrick, C. (2013). Child mental health, psychosocial well-being and resilience in Afghanistan: a review and future directions. In F. C. \& F. M. (Eds.), Handbook of resilience in children of war (pp. 51-79). New York: Springer.

White, J., Shelton, K. H., \& Elgar, F. J. (2014). Prospective associations between the family environment, family cohesion, and psychiatric symptoms among adolescent girls. Child Psychiatry and Human Development, 45(5), 544-554. https://doi.org/10. 1007/s10578-013-0423-5.

WHO. (2006). The World Health Report 2006: working together for health. http://www.who.int/whr/2006/en/.

WHO. (2017). Health statistics and information systems: definition of regional groupings. WHO regions. http://www.who.int/hea lthinfo/global_burden_disease/definition_regions/en/.

World Bank. (2017). World Bank Country and Lending Groups: World Bank list of economies. https://datahelpdesk.worldbank. org/knowledgebase/articles/906519-world-bank-country-andlending-groups.

*Yousafzai, Rasheed, Rizvi, Armstrong, \& Bhutta. (2014). Effect of integrated responsive stimulation and nutrition interventions in the Lady Health Worker programme in Pakistan on child development, growth, and health outcomes: a cluster-randomised factorial eff ectiveness trial. The Lancet, 384, 1282-1293. https:// doi.org/10.1016/S0140-6736(14)60455-4.

*Yousafzai, Rasheed, M. A., Rizvi, A., Armstrong, R., \& Bhutta, Z. A. (2015). Parenting skills and emotional availability: an RCT. Pediatrics, 135(5), e1247-e1257. https://doi.org/10.1542/peds. 2014-2335.

*Yu, J., Roberts, M., Shen, Y., \& Wong, M. (2015). Behavioral family therapy for Chinese preschoolers with disruptive behavior: a pilot study. Journal of Child and Family Studies, 24(5), 1192-1202. 